\title{
Elastic Shape Analysis of Brain Structures for Predictive Modeling of PTSD
}

This paper was downloaded from TechRxiv (https://www.techrxiv.org).

LICENSE

CC BY 4.0

SUBMISSION DATE / POSTED DATE

$13-09-2021 / 24-09-2021$

\section{CITATION}

Wu, Yuexuan; Kundu, Suprateek; Stevens, Jennifer S.; Fani, Negar; Srivastava, Anuj (2021): Elastic Shape Analysis of Brain Structures for Predictive Modeling of PTSD. TechRxiv. Preprint. https://doi.org/10.36227/techrxiv.16611916.v1

$\mathrm{DOI}$

10.36227/techrxiv.16611916.v1 


\title{
Elastic Shape Analysis of Brain Structures for Predictive Modeling of PTSD
}

\author{
Yuexuan Wu, Suprateek Kundu, Jennifer S. Stevens, Negar Fani, Member, IEEE, and Anuj Srivastava, \\ Fellow, IEEE
}

\begin{abstract}
Predictive modeling involving brain morphological features and other covariates is of paramount interest in such heterogeneous mental disorders as PTSD. We propose a comprehensive shape analysis framework representing brain substructures, such as the hippocampus, amygdala, and putamen, as parameterized surfaces and quantifying their shape differences using an elastic shape metric. Under this metric, we compute shape summaries (mean, covariance, PCA) of subcortical data and represent individual shapes by their principal scores under a shapePCA basis. These representations are rich enough to allow visualizations of full 3D structures and help understand localized changes. Subsequently, we use these PCs, the auxiliary exposure variables, and their interactions for regression modeling and prediction. We apply our method to data from the Grady Trauma Project (GTP), where the goal is to predict clinical measures of PTSD. The framework seamlessly integrates accurate morphological features and other clinical covariates to yield superior predictive performance when modeling PTSD outcomes. This approach reveals considerably greater predictive power under the elastic shape analysis than the current approaches and helps identify local deformations in brain shapes associated with PTSD severity.
\end{abstract}

Index Terms-Computational anatomy, elastic shape analysis, PTSD diagnosis, statistical regression models, shape PCA.

\section{INTRODUCTION}

$\mathbf{R}$ ECENT studies have increasingly documented the extent of traumatic experiences in our society $-60.7 \%$ of men and $51.2 \%$ of women experience at least one potentially traumatic event [1]. A significant proportion of these events occurs during young age; for example, $26 \%$ of males and $18 \%$ of females reported having experienced at least one traumatic event at a young age [2]. Of those experiencing traumatic events, $10-40 \%$ develop psychiatric symptoms of

Manuscript submitted September 13, 2021. This work was supported in part by the National Institutes of Health under Grant R01 MH120299 and in part by the National Science Foundation under Grant DMS 1953087. (Corresponding author: Yuexuan Wu.)

Yuexuan $\mathrm{Wu}$ and Anuj Srivastava are with the Department of Statistics, Florida State University, Tallahassee, FL 32306 USA (email:yw17g@my.fsu.edu).

Suprateek Kundu is with the Department of Biostatistics and Bioinformatics, Emory University, Atlanta, GA 30322 USA.

Jennifer S. Stevens and Negar Fani are with the Department of Psychiatry and Behavioral Sciences, Emory University, Atlanta, GA 30322 USA.

The Supplementary Materials are available online at https://github.com/wuyx5/Elastic-Shape-Analysis-PTSD clinical relevance [3], such as post-traumatic stress disorder (PTSD). PTSD is the fourth most common mental disorder in the USA and results in significant impairments of psychological and physical health [1]. Diagnosing psychiatric disorders using brain imaging data has started gaining attention in the literature. Imaging provides physical biomarkers, such as volumes and shapes of anatomical structures in the human brain. Subcortical structures, in particular, have been implicated in preclinical neuroscience research in behaviors and phenotypes with crucial relevance to mental health.

From a methodology perspective, Voxel-based morphometry (VBM) is used commonly to identify associations between PTSD and various brain regions. These regions include the amygdala, prefrontal cortex, temporal cortex, insula, thalamus, anterior cingulate cortex (ACC), and hippocampus [4], [5]. For example, Chen et al. [6] reported PTSD-related alterations in hippocampus, resulting in lower volume of the CA1 sub-field . In adult military veterans, PTSD has been linked with smaller para laminar and lateral subnuclei, but larger central, medial, and cortical nuclei [7]. In contrast, in youth exposed to a terror attack, PTSD symptoms were associated with smaller volumes across all significant subnuclei [8]. These findings suggest that the developmental timing and type of trauma exposure may affect neural phenotypes in PTSD. However, the analyses of subnuclei are limited by tissue contrast and spatial resolution available in typical 3T MRI research scans. Thus, alternative methods for incorporating morphology of subcortical regions are needed to provide critical neural biomarkers of various psychiatric conditions.

While the relationships between morphologies of brain structures, such as hippocampus and putamen, and some disorders, such as ADHD, Alzheimer's, and Schizophrenia, are well established [9], [10], the literature on PTSD is relatively limited. Note that the value of structural analysis lies in its ability to reveal local regions of variation. The addition of localized geometrical descriptions allows the detection of subtle changes in a surface. This signal may get lost when averaged across the whole ROI. For example, although hippocampal changes are expected between PTSD and control groups, some volumetric analyses in [11], [12] failed to find significant differences in hippocampi of the normal and diseased groups. In contrast, full shape analysis can allow investigators to detect regions of equal but opposite variation within a single surface, which would have otherwise been canceled out had they been reduced to a single, scalar value. Furthermore, despite recent 
developments in the use of brain structures for assessing PTSD severity, there are significant unanswered questions. They relate to how alterations in the brain structure and shape after trauma exposure result in PTSD onset and progression.

In this article, we apply elastic shape analysis method [13] to analyze brain subcortical structures. It characterizes shapes as parameterized surfaces in $\mathbb{R}^{3}$, instead of a point sets, and it incorporates dense registration of points across objects. The elastic shape analysis quantifies pure shape variability, modulo shape-preserving transformations, and helps discern subtle variations across populations by minimizing mis-registration errors. The elastic shape analysis helps align, register, and compare shapes of surfaces. It also provides shape summaries, i.e., statistical means and covariances of shapes sampled from a population. The computation of covariance leads to principal component analysis (PCA) of sample shapes and the set of principal coefficients (PC) forms a parsimonious, lowdimensional representation of those brain structures. By including interactions between a subset of PCs and confounding variables (such as trauma), the proposed approach provides a parsimonious and flexible classification or prediction approach not restricted to vertex-wise analysis. It enables non-linear associations between the brain shape, potential confounders, and their interactions with the clinical phenotype of interest. This results in superior prediction performance compared to the state-of-the-art shape analysis methods in PTSD literature.

An essential strength of these shape features is reconstructing full shapes from these feature vectors (principal components). Consequently, one can visualize changes in the shapes of a structure by varying only one or multiple features at a time. This provides a vital tool for physicians and clinicians to visualize localized changes or deformations in the brain anatomy for statistically significant shape features or principal components. We focus on a pre-specified subset of subcortical structures and their interactions with demographic and exposure confounding variables to classify and predict PTSD severity. In contrast to most existing studies that focus on brain shape changes in military veterans, our study is one of the first to investigate brain shape changes in PTSD in conjunction with co-morbidities such as trauma in a civilian minority population of AA females.

The main contribution of this paper are:

1) The use of elastic shape analysis to characterize shapes of subcortical structures as parameterized surfaces. This framework integrates the registration of surfaces as a part of shape analysis, and provides a comprehensive toolbox for registering, comparing, summarizing, and testing shapes. This leads to a representation of shapes using (invertible) PCA features.

2) The use of shape features and other clinical covariates in statistical regression models for modeling PTSD severity measures as response variables. This results in shortlisting and analysis of features that are significant in predicting PTSD.

3) The visualization of local deformations associated with significant morphological features and their interpretations in predicting PTSD severity.

\section{Materials And Methods}

This section lays out the entire pipeline for extracting and analyzing shapes of subcortical brain surfaces. This pipeline is illustrated pictorially in Fig. 1 with the time costs computed on a laptop with Intel i7-8705G processor. As the figure shows, the proposed pipeline has three main steps: (i) pre-processing of the original data; (ii) registration and shape analysis of 3D surfaces; and (iii) regression models for analysis of PTSD. We describe these steps next, starting with introducing the data used in the experiments presented later.

\section{A. Data Description}

In this study, we utilize T1-weighted MRI scans of brains of 90 subjects. The dataset also contains demographic information with the questionnaire results on PTSD symptoms and traumatic experiences.

T1-weighted MRI scans: The original data is T1-weighted MRI scans acquired by Emory University Grady Trauma Project using Siemens Tim Trio [14]. Field of view is $224 \mathrm{~mm} \times 256 \mathrm{~mm}$, while repetition time and echo time are $2600 \mathrm{~ms}$ and $3.02 \mathrm{~ms}$ separately.

Demographic Information: Participants of the experimental data collection are all African American women. The other demographic information included in the data is: age (18-61), education $(0-5)$, employment $(0,1)$ and disability $(0,1)$.

Questionnaire Results: In the study, we are most interested in participants' answers to questions related to PTSD symptoms and traumatic experiences. Specifically, we focus on three questionnaire results: (i) PTSD Symptom Scale (PSS), which measures the presence and frequency of current PTSD symptoms and has a range of 0-42; (ii) Childhood Trauma Questionnaire Total Score (CTQTOT), which is a 25-item inventory of different types of childhood maltreatment including abuse and neglect and has a range of 25-125; and (iii) Beck Depression Inventory (BDI), which is a 21-question multiplechoice self-report inventory and has a range of 0-63.

\section{B. Data Pre-processing}

Here we describe the steps for extracting subcortical structures from brain imaging data. We use the state-of-art packages to pre-process the MRI scans obtained as the original data. We first convert 176 DICOM scan files for each subject into a single NIfTI file using SPM12 [15]. The NIfTI images each have a resolution of $240 \times 256 \times 176$.

Next, we utilize the FMRIB Software Library (FSL) that contains image analysis and statistical tools for functional, structural and diffusion MRI brain imaging data. Among the tools in FSL, FSL FIRST [16] is a model-based segmentation/registration tool. FSL FIRST can segment a T1-weighted MRI image into 15 subcortical structures' surfaces. Using some manually segmented images, in which the subcortical labels are parameterized as surface meshes and modeled as a point distribution model, FSL FIRST trains an automatic 


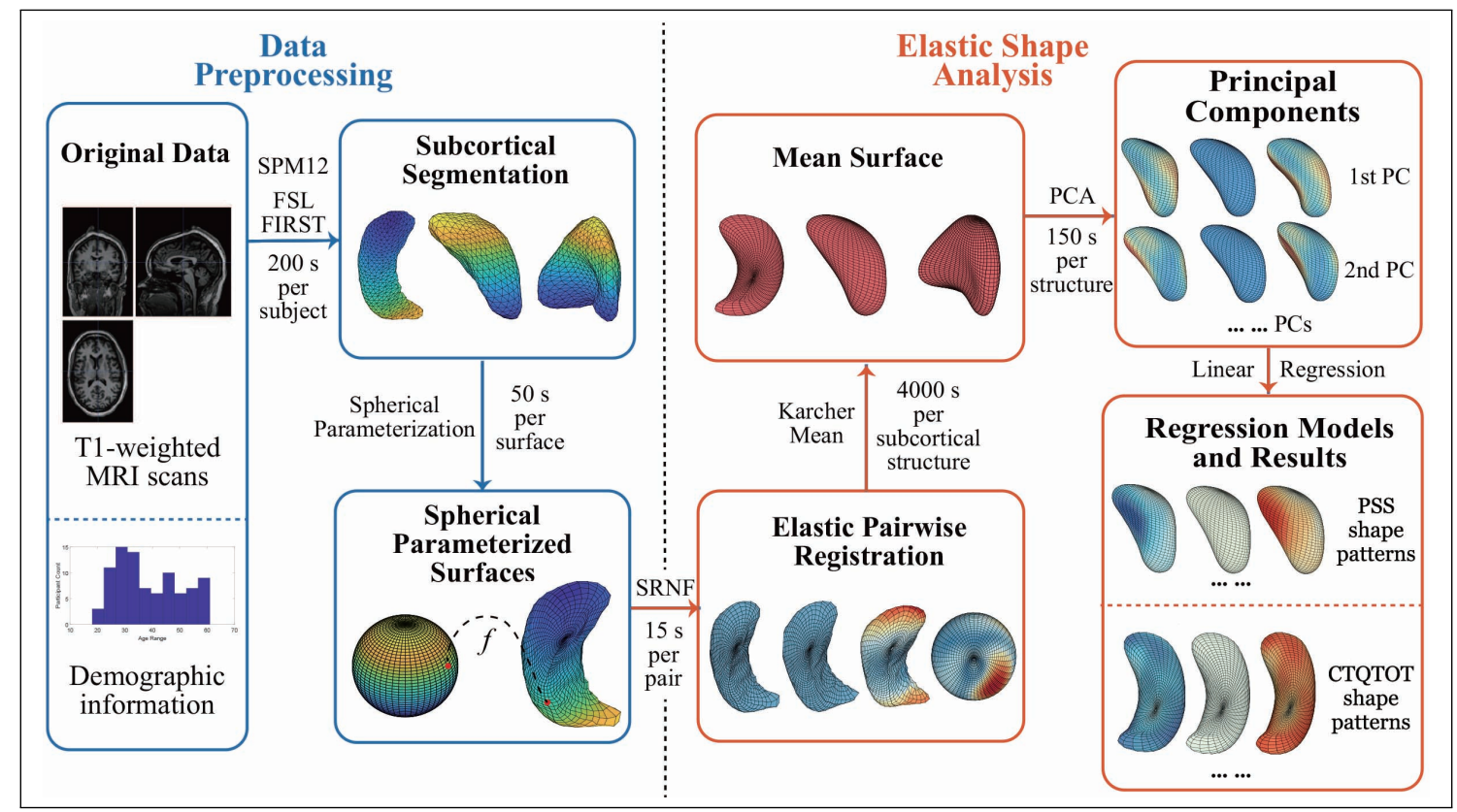

Fig. 1: Pipeline steps and time cost. The time cost is computed with Intel i7-8705G. SRNF: square root normal field, PCA: principal component analysis, PC: principal component, PSS: PTSD symptom scale, and CTQTOT: childhood trauma questionnaire total score.

segmentation model using a Bayesian approach. The inputs of FSL FIRST are T1-weighted MRI images in NIfTI file format, and the outputs are triangular meshed surfaces of 15 subcortical brain structures. Although there are several structures available for study, this paper mainly focuses on three structures: left hippocampus, left amygdala and left putamen. The outputs of FSL FIRST are $m \times 3$ vertex coordinates and $(2 m-4) \times 3$ edges. In this data, $m$ is 732 for hippocampus surfaces and 642 for all other subcortical structures.

We then apply a spherical conformal mapping and Tuette mapping algorithms in [13] to transform the triangulated meshes into spherically-parameterized surfaces. The method first creates progressively finer mesh structure with triangles, and then embeds the mesh vertices into a sphere. The surfaces are spherically parameterized since each point on the surface corresponds uniquely to a point on the unit sphere $\mathbb{S}^{2}$. This provides a representation of the surface as an embedding: $f$ : $\mathbb{S}^{2} \rightarrow \mathbb{R}^{3}$. Figure 2 illustrates the process: Column (a) shows an example of triangular mesh surface, column(b) and (c) are sphere $\mathbb{S}^{2}$ and the corresponding spherically-parameterized surface with $51 \times 51$ mesh. Points in the same color indicate the corresponding relationship.

\section{Framework: Elastic Shape Analysis}

To analyze shapes of subcortical structures and to discern shape changes with traumatic experiences, we utilize the elastic shape analysis approach, developed in the book [13]. This comprehensive theory provides several tools for analyzing shapes of 3D objects, including (1) metric for quantifying differences in their shapes, (2) deforming objects into each other using geodesic paths, (3) optimally registrating points across surfaces being compared, and (4) computing mean,

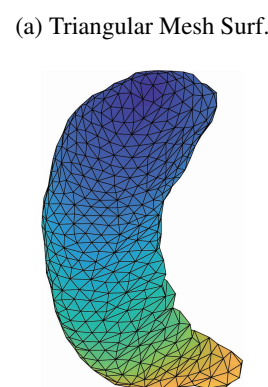

(b) Unit Sphere

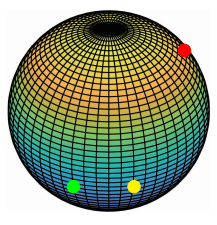

(c) Spherical Parameterized Surf.

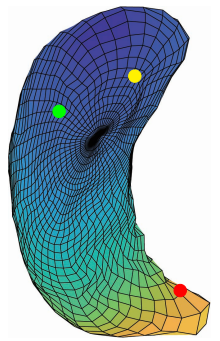

Fig. 2: Object pre-processing: Triangular mesh surface has $732 \times 3$ vertices and spherical parameterized surface has a $51 \times$ 51 grid. Each vertex on the spherical parameterized surface corresponds to a vertex on the $51 \times 51$ mesh unit sphere $\mathbb{S}^{2}$.

covariance, and PCA of shapes. An important aspect of this approach is that these results are theoretically invariant to the chosen shape-preserving transformations (rigid motions, global scaling, and parameterizations of surfaces). From a practical perspective, it helps remove misregistration errors from the analysis. In the past, this framework has been applied to shape analysis of human bodies [17] and brain morphology associated with Alzheimers [9] and ADHD [10].

1) Elastic Metric and SRNF: Next, we present some salient ideas of this approach. The subcortical objects are considered as closed surfaces in $\mathbb{R}^{3}$. Each closed surface can be represented in a parametrized form using a smooth map: $f: \mathbb{S}^{2} \rightarrow \mathbb{R}^{3}$. Let $\mathcal{F}$ denote the space of all such surfaces. If $s=(u, v)$ is a point on the sphere $\mathbb{S}^{2}$, then the partial derivatives $f_{u}$ and $f_{v}$ denote two orthogonal tangent vectors to the surface $f$ at the point $f(s)$. The (unnormalized) normal vector at point $s$ is given by $\mathbf{n}(s)=f_{u} \times f_{v}$, where $\times$ 
indicates the cross product in $\mathbb{R}^{3}$. Figure 3 shows a surface $f$, parametrized by points on a unit sphere $\mathbb{S}^{2}$, with the tangent and normal vectors at point $f(s)$ on the surface.

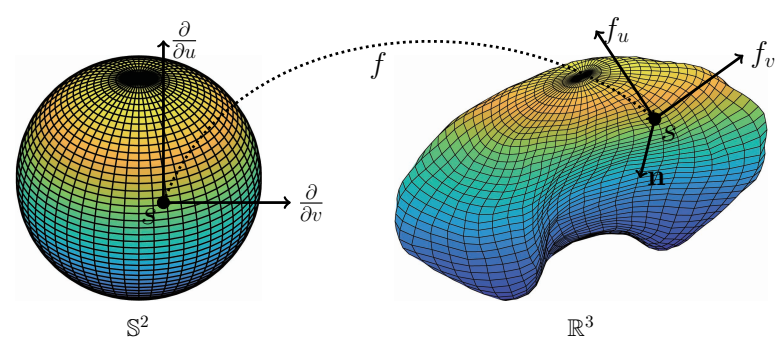

Fig. 3: Local geometry of a parameterized surface viewed as a mapping $f: \mathbb{S}^{2} \rightarrow \mathbb{R}^{3}$. The figure shows the tangent vectors $f_{v}$ and $f_{u}$ and the normal vector $\mathbf{n}$ at point $f(s)$ on $f$.

Let $\Gamma$ be the set of all orientation-preserving diffeomorphisms of $\mathbb{S}^{2}$; the elements of $\Gamma$ help us re-parameterize surfaces. For any parameterized surface $f \in \mathcal{F}$ and a $\gamma \in \Gamma$, the composition $f \circ \gamma$ denotes a re-parameterization of $f$. Equivalently, elements of $\gamma$ also help in a dense registration of points across two surfaces, say $f_{1}$ and $f_{2}$. Initially, for any $s \in \mathbb{S}^{2}$, the point $f_{1}(s)$ on $f_{1}$ is said to be registered to the point $f_{2}(s)$ on $f_{2}$. However, if we re-parameterize $f_{2}$ by $\gamma$, then the point $f_{1}(s)$ is now registered to the point $f_{2}(\gamma(s))$ on $f_{2}$. Thus, $\gamma$ becomes a tool for controlling the registration between $f_{1}$ and $f_{2}$. The next question is: How can we find the best registration between any two surfaces $f_{1}$ and $f_{2}$ ? A related question is: What should be the objective function for defining and calculating the optimal $\gamma$ that best registers $f_{2}$ with $f_{1}$ ? An obvious choice would be the $\mathbb{L}^{2}$ norm, but it is degenerate and leads to singularities in solutions. While one can impose additional penalties to avoid degeneracy, the resulting solution is not inverse symmetric. That is, the registration of surface $f_{1}$ to $f_{2}$ may not be consistent with the registration of surface $f_{2}$ to $f_{1}$. From a mathematical perspective, the problems in using the $\mathbb{L}^{2}$ norm for registering surfaces stem from the following fact. In general, for any $f_{1}, f_{2} \in \mathcal{F}$ and $\gamma \in \Gamma$, we have: $\left\|f_{1}-f_{2}\right\| \neq\left\|f_{1} \circ \gamma-f_{2} \circ \gamma\right\|$. In the other words, we lose some information about the shape of the surfaces after reparameterization if we use the $\mathbb{L}^{2}$ distance to compare them.

A better alternative for registration and shape analysis comes from an elastic Riemannian metric. While this metric's original form is too complex for practical usage, a squareroot representation of surfaces simplifies their usage. This representation, termed the square root normal field (SRNF), is defined as follows: for $s \in \mathbb{S}^{2}$, define $q(s)=n(s) /|n(s)|^{\frac{1}{2}}$, where $n(s)$ is the normal at a point $f(s)$ as explained earlier. Thus, $q$ is nothing a but a normal vector field on the surface $f$ with the magnitude given by $\sqrt{|n(s)|}$. If $q$ is the SRNF of a surface $f$, then the SRNF of the re-parameterized surface $f \circ \gamma$ is given by: $(q \star \gamma)(s) \equiv Q(f \circ \gamma)(s)=\sqrt{\mathbf{J}[\gamma(s)]}(q \circ \gamma)(s)$, where $\mathbf{J}[\gamma(s)]$ is the Jacobian of $\gamma$ at $s$. One of the fundamental advantages for using SRNF representation is the following. Let $q_{1}$ and $q_{2}$ denote SRNFs of two surfaces $f_{1}$ and $f_{2}$, respectively. Then, the $\mathbb{L}^{2}$ norm between SRNF satisfies the condition $\left\|q_{1}-q_{2}\right\|=\left\|O\left(q_{1} \star \gamma\right)-O\left(q_{2} \star \gamma\right)\right\|$ for all 3D rotations $O \in S O(3)$ and all $\gamma \in \Gamma$. This property allows us to define a shape metric between $f_{1}$ and $f_{2}$ according to:

$$
d_{s}\left(f_{1}, f_{2}\right)=\inf _{(O, \gamma) \in S O(3) \times \Gamma}\left\|q_{1}-O\left(q_{2} \star \gamma\right)\right\| .
$$

In other words, we fix one of the surfaces and find the best rotation and re-parameterization of the second surface so as to minimize this distance. Note that if we apply an arbitrary rotation or re-parameterization to either $f_{1}$ or $f_{2}$ or both, their shape distance remains unchanged!

The computation of shape metric in (1) requires solving for the optimal $O^{*} \in S O(3)$ and $\gamma^{*} \in \Gamma$. We use the Procrustes method to solve for the optimal rotations of $f_{1}$ while keeping $\gamma \in \Gamma$ fixed, and we use a gradient-descent to optimize over $\Gamma$, whose details and algorithms are presented in [13], while keeping the rotation fixed. After finding the optimal $O^{*} \in$ $S O(3)$ and $\gamma^{*} \in \Gamma$, and applying them to surface $f_{2}$ constitutes surface registration and alignment. The aligned surface is $f_{2}^{*}=$ $O^{*}\left(f_{2} \circ \gamma^{*}\right)$, and the distance between $f_{2}^{*}$ and $f_{1}$ is the shortest among all $O\left(f_{2} \circ \gamma\right)$ for any $O \in S O(3), \gamma \in \Gamma$.

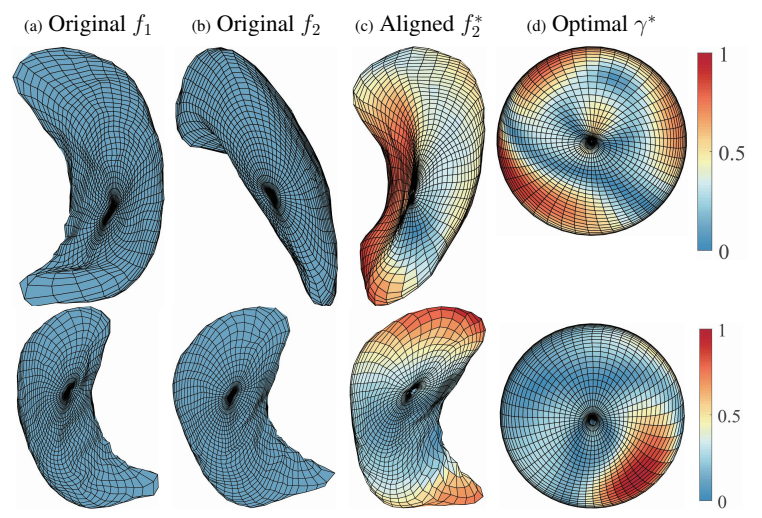

Fig. 4: Two examples of elastic surface alignment and registration. In each row: (a) the original template surface $f_{1}$; (b) the original surface $f_{2}$ to be aligned; (c) the optimal re-parametrized surface $f_{2}^{*}$; and (d) the optimal reparameterization function $\gamma^{*}$ shown on the unit sphere. Colors indicate the relative determinant of the Jacobian of repatameterization function.

Figure 4 illustrates two examples of pairwise surface registration, where $f_{2}$ is registered with $f_{1}$. Columns (a) and (b) show the original $f_{1}$ and $f_{2}$, and column (c) shows $f_{2}^{*}$, the surface after optimal re-parameterization and rotation. The corresponding re-parameterization function $\gamma^{*}$ is shown in column(d), where the color indicates the relative determinant of the Jacobian $\operatorname{det} \mathbf{J}(\gamma)$ of re-parameterization function $\gamma^{*}$. The first example is a left putamen surface registered with a left hippocampus surface, and the second example is the pairwise elastic registration of two left hippocampus surfaces. In the first example, we can see that the left putamen's two outermost parts are registered with anterior and posterior ends of the left hippocampus, respectively. The second example shows that the two surfaces' anterior, middle and posterior parts are well-aligned, making it possible to compare two surfaces' shapes. Since the two surfaces are more different 
in the anterior end and posterior end, re-parameterization acts more prominently in these parts.

2) Elastic Registration: A Simulation Study: Here, in order to illustrate and validate the necessity of surface registration, we conduct some simulation studies. We randomly generate 40 simulated surfaces using PCA representations of shapes of left hippocampus (the use of PCA is detailed later in this paper). More specifically, we use only the first principal direction $v_{1}$ in this experiment and generate 20 surfaces each on either side of mean $\mu$ along that direction. That is, we generate $f_{i}=$ $\mu+x_{i} v_{1}, i=1,2, \ldots, 40$, where $x_{i} \in(0,1]$ for $I \leq 20$ amd $x_{i} \in[-1,0)$ for $i>20$.

Since PCA is performed after surface registration, these simulated surfaces can be considered well aligned and registered. We calculate the pairwise distances between surfaces as $d_{i j}=\left\|f_{i}-f_{j}\right\|$, where $i, j=1,2, \ldots, 40$. (a) and (b) in Fig. 5 show the heat map and multidimensional scaling (MDS) plot of the distances between surfaces. First 20 surfaces are presented by blue dots and last 20 are presented by red dots. This figure illustrates that for registered surfaces, the shapes that are on the same direction of principal shape component have relatively small distance, and are correctly clustered into the same class. (a) Heat Map-Registered

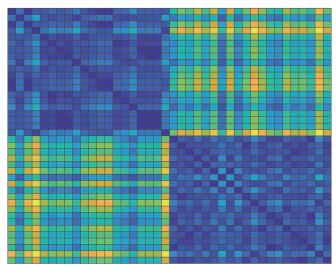

(c) Heat Map-Not Registered

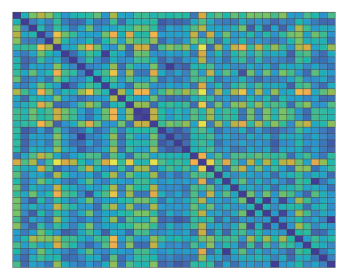

(b) MDS Plot-Registered

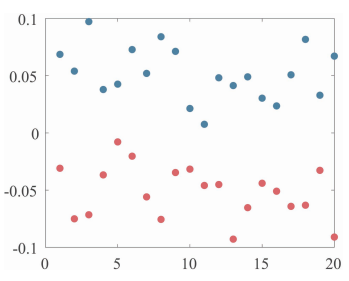

(d) MDS Plot-Not Registered

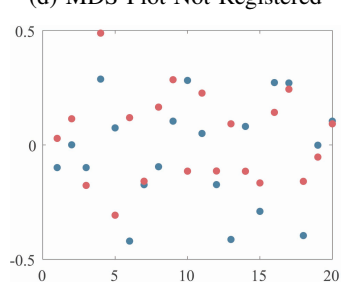

Fig. 5: (a) Heat map and (b) MDS plot of distances between well-aligned and registered surfaces. (c) Heat map and (d) MDS plot of distances between randomly-parameterized surfaces (not well registered). Colors of the heat map indicates the relative distances between surfaces. Blue dots in MDS plot indicate the first 20 simulation surfaces and red dots indicate the last 20 simulation surfaces.

Next, we introduce random parameterization functions $\gamma_{i} \in$ $\Gamma$ and apply $\gamma_{i}$ 's to $f_{i}$ 's to simulate randomly parameterized surfaces. For each $i$, the surface $\tilde{f}_{i}=f_{i} \circ \gamma_{i}$ has the same shape as $f_{i}$, but a different parameterization. The distances between unregistered surfaces are again calculated by $\tilde{d}_{i j}=\left\|\tilde{f}_{i}-\tilde{f}_{j}\right\|$. (c) and (d) in Fig. 5 shows the heat map and MDS plot of the distances between randomly parameterized surfaces. We see that distances between surfaces that have similar shapes are not smaller anymore and MDS plot shows that unregistered surfaces are not effectively clustered.

3) Shape Analysis Tools: Geodesics, Mean, and PCA: The framework developed so far allows for representing and registering anatomical surfaces and comparing shapes of these surfaces pairwise using a proper shape metric. This metric is used to develop some additional statistical tools, leading to a compact way of representing shapes. These tools include finding geodesics between shapes, computing means of shapes of surfaces, and discovering principal modes of shape variation in a given set of shapes.

Shape Geodesic: Given two surfaces, $f_{1}$ and $f_{2}$, a geodesic between their shapes is a visualization of the optimal deformation from one to the other. Although there are more sophisticated ways to compute exact geodesics, we use a simple linear interpolation to approximate this deformation according to: $\alpha_{\tau}^{*}(s)=(1-\tau) f_{1}(s)+\tau f_{2}^{*}(s), \quad s \in \mathbb{S}^{2}$, where $\tau \in[0,1]$ is the time index of the geodesic. At $\tau=0$ we have alpha $=f_{1}$ and at $\tau=1$ we have alpha $=f_{2}^{*}$. Figure 6 shows two examples of geodesics, where the upper one is the geodesic between unregistered surfaces and the lower one is between elastically registered surfaces. Due to the misalignment of points between surfaces, the hippocampus's posterior end "degenerates" on the upper geodesic at the midway point. In contrast, the anatomical features of hippocampus surfaces are preserved with elastic registration, making the midway surfaces along the geodesic more interpretable.

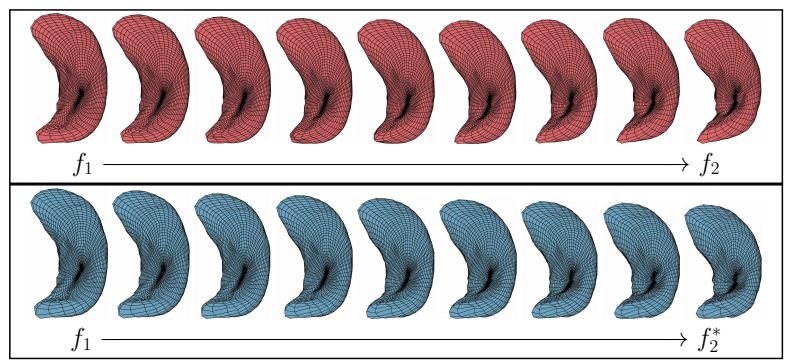

Fig. 6: Two examples of geodesic between left hippocampus surfaces. Upper row: between unregistered surfaces; lower row: between elastic registered surfaces.

Additional examples of elastic geodesics can be found in Supplementary Fig. 1. We also provide these deformations as GIF files in the Supplementary Materials.

Mean Shape: The next concept we introduce is the mean of a shape of shapes of surfaces $f_{1}, f_{2}, \ldots, f_{n}$. We define an intrinsic mean, or the Karcher mean as the shape $\mu$ that minimizes the sum of square of distances (under the shape metric) to the given shapes. That is, $\mu=\operatorname{argmin}_{f \in \mathcal{F}} \sum_{i=1}^{n} d_{s}\left(f, f_{i}\right)^{2}$. We use an iterative algorithm to compute this mean shape. Here we start by selecting an arbitrary surface as the initial guess for $\mu$. Then, in each iteration, we register each $f_{i}$ with the current mean and compute the (Euclidean) mean of these registered $f_{i} \mathrm{~s}$ in $\mathcal{F}$. Once the algorithm has converged, we obtain the mean shape $\mu$. We outline these steps using Algorithm 1. 


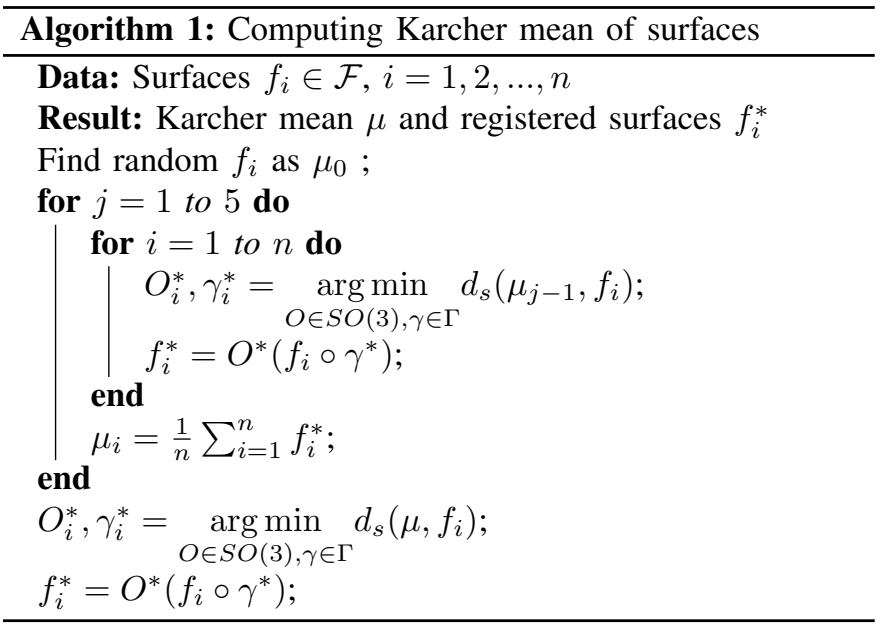

Shape PCA: Next, we perform Principal Component Analysis (PCA) to capture essential shape variability in a given set of surfaces. We start by computing the covariance matrix $C$ for surfaces: $C=\sum_{i=1}^{n} V_{i} V_{i}^{T}, \quad$ where $V_{i}=\operatorname{vec}\left(f_{i}-\mu\right)$, where vec denotes vectorization of a matrix. By performing singular value decomposition (SVD) on the covariance matrix $C$, we obtain the left singular vectors as the columns of the unitary matrix $U$. These columns form the principal directions of shape variability the data. This decomposition also results in singular values that indicate the variance of the shape variability among each of the principal directions.

Low-Dimensional Shape Representations: We use PCA to derive low-dimensional representations of shapes of objects for use in statistical models and regressions. During experiments, we randomly divide all surfaces (of a specific type, say hippocampus) into training and test groups. Then we compute the principal components of shape variation using only the training surfaces. Then, we compute the principal score for all test surfaces. For a test surface $f_{i} \in \mathcal{F}, i=1,2, \ldots, n$ and principal directions $U(:, d), d=1,2, \ldots, n$, the principal score is computed by: $z_{i, d}=\left\langle f_{i}-\mu, U(:, d)\right\rangle$. In this way, a highdimensional object $f_{i}$ is now represented by a $d$-dimensional vector $z_{i} \in \mathbb{R}^{d}$. It is important to note that this representation is invertible and we can reconstruct test surfaces according to: $\hat{f}_{i}=\mu+\sum_{d=1}^{n} z_{i, d} U(:, d)$. We validate this representation by examining the difference between the reconstructed surface $\hat{f}_{i}$ and the original surface $f_{i}$.

\section{Regression Model Design}

Having obtained low-dimension mathematical representations of subcortical structures' shapes, we can study their roles in statistical models for diagnosing PTSD. Specifically, we train ten linear models to test the significance of shape differences between subcortical surfaces in different childhood traumatic experience levels and PTSD symptom scales. In model 1 and model 5, we include the interactions between shape (for $d=5$ principal scores) and confounding variables (age and BDI). We design models 3, 4, 7, and 8 to test the interpretation power of shape in PTSD and experience levels. We use models 9 and 10 that include the intracranial volume (ICV) as a control covariate. Table I shows the design (predictors and responses) for ten different models. To capture the shape differences while minimizing noise, we take only the first 15 principal scores (PS) for each surface. These represent the 15 most dominant modes of shape variation to train the model. In these models, we select the most significant predictors using bidirectional stepwise regression.

\section{E. Comparison with Vertex-wise Analysis}

To verify the effectiveness and ability of elastic shape analysis in identifying shape differences attributed to PTSD disease, we compare it with vertex-wise analysis, a widely applied shape analysis method in neuroimaging. We perform this comparison by applying a similar pipeline to vertex-wise surfaces, as our elastic approach, and comparing the results.

We start by representing surfaces using sets of vertices or point clouds. Next, we apply the state-of-art point cloud registration algorithm, iterative closest point (ICP) [18], to register individual surfaces. This step is analogous to the elastic registration step. After registration, we compute mean, covariance and PCA, in the same way as elastic shape analysis. We compare the two methods - elastic shape analysis and vertex-wise analysis - using (i) average inter- and intra-class total point-wise distances; and (ii) regression model results with the same model design and different principal scores of each analysis method. Here we define PTSD diagnosis, a binary ( 0 and 1) label included in the original data, as the class label for a subject. Although this PTSD diagnosis data is obtained using thresholding and cannot be considered the original medical diagnosis, it is still acceptable to use it as a label for comparing methods. We compute average inter- and intra-class total point-wise distance according to:

$$
d_{\text {inter(intra) }}=\frac{\sum_{i, j=1, j>i, l_{j} \neq(=) l_{i}}^{90}\left\|\mathbf{x}_{j}-\mathbf{x}_{i}\right\|}{\#\left\{(i, j) \mid j>i, l_{j} \neq(=) l_{i}\right\}},
$$

where $\mathbf{x}_{i, k}=\left(x_{i, k}, y_{i, k}, z_{i, k}\right)$ and $\mathbf{x}_{j, k}=\left(x_{j, k}, y_{j, k}, z_{j, k}\right)$ are $x, y$ and $z$ coordinates of the $k t h$ point (with $K$ points in total) of the $i t h$ and $j t h$ surfaces. Recall that we have 90 surfaces in total for each subcortical structure, so $i, j=1, \ldots, 90$. Also, here $l_{i}$ and $l_{j}$ are the surface class labels (0 or 1) for the $i t h$ and $j$ th surfaces separately. We compute both average interand intra-class total point-wise distances for the two methods after their respective registrations.

\section{Results}

This paper focuses on discovering the impact of traumatic experiences and PTSD disease on three subcortical brain structures surface: left hippocampus, left amygdala, and left putamen. Thus, we present the shape analysis results for three groups of surfaces in relation with traumas and PTSD disease.

\section{A. Shape Statistics of Surfaces}

We start by presenting the shape statistics, including geodesics, Karcher mean, principal components and reconstruction accuracy for three subcortical structures separately. 
1) Karcher Mean: First, we present the Karcher mean surfaces $\mu$ as computed with Algorithm 1 for different structures. Figure 7 shows some registered individual surfaces $f_{i}^{*}$ (drawn around the mean) and their Karcher mean surfaces $\mu$ (drawn in the middle). Column (a), (b), (c) illustrate the left hippocampus, left amygdala and left putamen, respectively. We see that the Karcher mean surfaces capture salient anatomical shape features among the groups while reducing the individual noise.

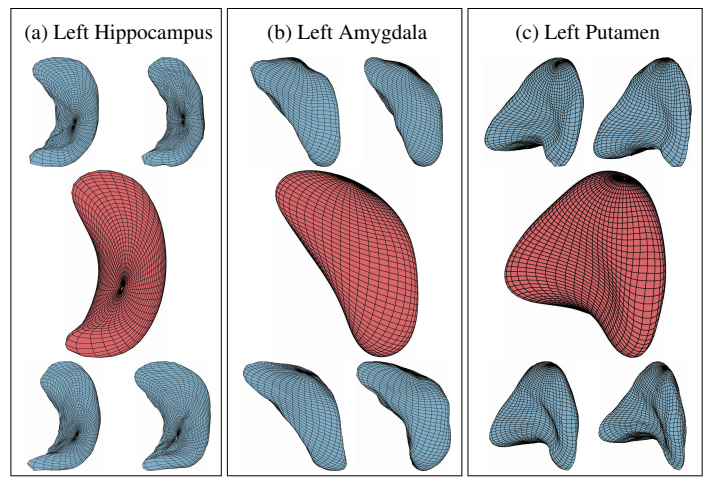

Fig. 7: Karcher mean surfaces and sample individual surfaces of three subcortical structures. Surrounding blue ones are randomly selected sample individual surfaces, and the middle red ones are Karcher mean surfaces computed using Algorithm 1.
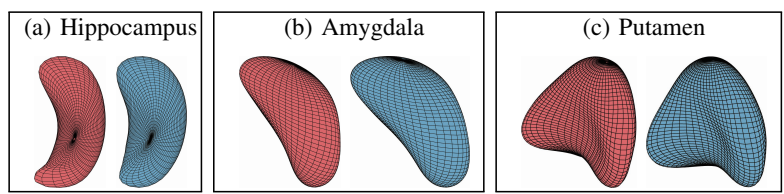

Fig. 8: Comparison between mean surfaces computed with and without elastic registration. Red ones are mean surfaces computed using Algorithm 1 with surface registration, while blue ones are computed without surface registration.

2) Principal Components: Using the mean surfaces $\mu$, we can now compute covariance matrix and perform PCA. We present the PCA results for three subcortical structures separately. Figure 9 illustrates the 1st principal component of left hippocampus, left amygdala and left putamen surface shape. We show a principal direction using the elastic deformation path $\mu-\sigma \longrightarrow \mu \longrightarrow \mu+\sigma$. Colors on a surface indicate the patch-wise shape differences of that surface when compared with the mean surface. Note that along the 1st principal component: (a) for left hippocampus, the largest shape variability is in the angle of the posterior endings; (b) for left amygdala, the surfaces "bends" more towards the "tail" end; and (c) for left putamen, the curvature of the middle part changes.

The 2nd and 3rd principal components of three subcortical structure surfaces are shown in Supplementary Figs. 2, 3, and 4. We also provide interactive slider graphs to help visualize changes along different principal components for these subcortical structures in the Supplementary Material.

We can quantify the level of shape variability explained by the principal components using the cumulative proportion of total singular values, as shown in Fig. 10 for both vertex-wise

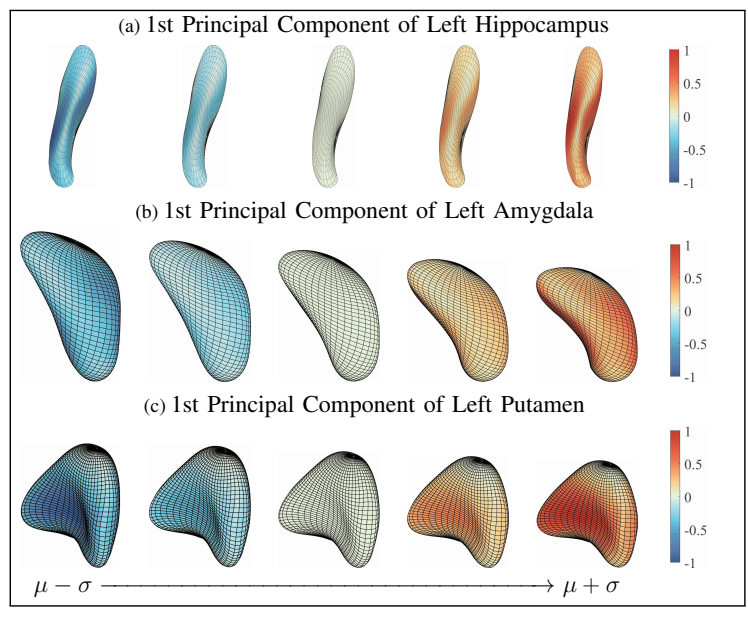

Fig. 9: 1st principal components of three subcortical structures. The figures show the deformation along the path of $\mu-\sigma \rightarrow \mu+\sigma$ following 1 st principal direction. Color indicates the small patch's relative shape difference (deformation level) compared with the mean surface.

analysis and elastic shape analysis. Under elastic shape analysis, the 1st principal component explains about 33\%, 37\%, and $42 \%$ variability for the left hippocampus, left amygdala, and left putamen, respectively. For these three structures, we can explain over $95 \%$ of the variability in shapes with 14,15 , and 10 principal components in total, respectively. Therefore, we will use the first 15 principal components to represent a shape in the subsequent regression analysis. Furthermore, when comparing elastic shape analysis (red lines) with vertexwise analysis (blue lines), we conclude that elastic shape analysis explains more shape variability with the same number of principal components.
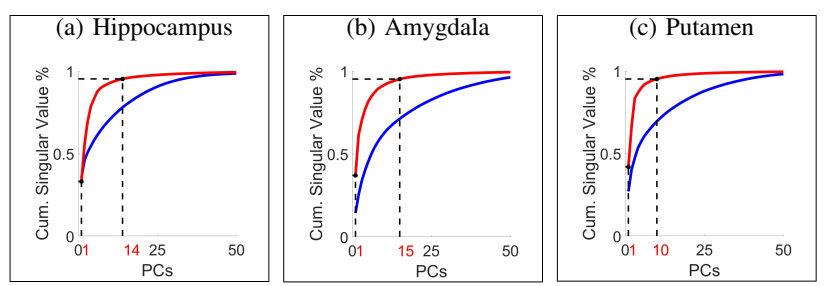

Fig. 10: The cumulative proportion of total singular values of the covariance. Red lines: elastic shape analysis; blue lines: vertex-wise analysis. Under elastic shape analysis, the 1st PCs can explain about 33\%, 37\% and $42 \%$ variability of surface shape for left hippocampus, amygdala and putamen. The cumulative singular values take up more than $95 \%$ of total singular values with 14,15 and 10 PCs respectively.

An important strength of this shape representation is that we can map these features back to the object space. Figure 11 presents some examples of such reconstructions. We use $90 \times 0.8=72$ surfaces to compute principal components and the other 18 surfaces to reconstruct and test. The right column shows sample individual surfaces for each subcortical structure, and the left side shows surfaces reconstructed using $f_{i}=\mu+\sum_{j=1}^{d} x_{i, j} v_{j}$. The surfaces are reconstructed with 
$d=0,1,5,15$, and 72 principal components added to the mean surface respectively. Color indicates the patchwise shape differences between reconstructed surface and example surface $\left|\hat{f}_{i}-f_{i}\right|$. As more and more principal components added, the patches change color from red to blue, and the shapes of reconstructed surfaces look similar to the sample surfaces. When $d=72$ principal components (all of the principal components) are used, the reconstructed is almost identical to the original surface. The reconstructed surfaces are mostly blue, which means the difference between reconstructed surface and example surface is relatively very small. This result illustrates that elastic mean and PCA successfully capture the modes of shape variations in subcortical structure surfaces, and represent individual shapes using a small number of PCA coefficients.

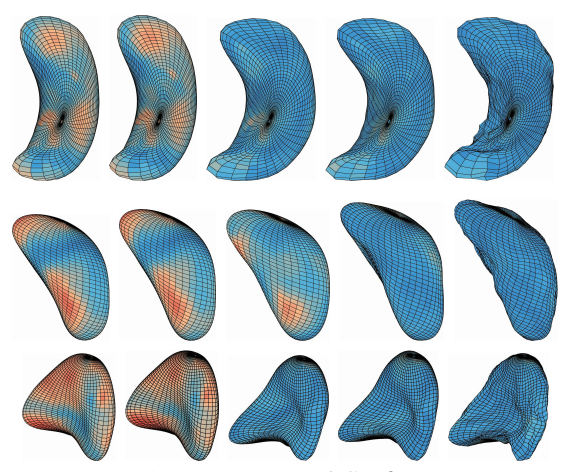

(a) Reconstructed Surfaces

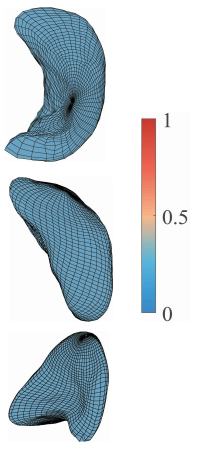

(b) Target
Fig. 11: Sample surface reconstructions of three subcortical structures. (a) are reconstructed surfaces with first $0,1,5,15$ and 72 principal components. (b) are target surfaces. Color indicates the small patch's relative shape difference compared with the target surface.

We provide GIF examples of the surface reconstruction in the Supplementary Material.

\section{B. Regression Models}

Next, we use these compact shape representations as predictors in regression models. Specifically, we study the ten linear regression models listed in Table I. Table I shows the adjusted $R^{2}$ for the fitted models, and Tables II and III list the significant principal components with their signs of regression coefficients and the corresponding $p$-values and significant interaction terms for each model.

TABLE I: Adjusted $R^{2}$ of Regression Models

\begin{tabular}{c|c|c}
\hline Model\# & Model Design & $R^{2}$ \\
\hline 1 & PSS $\sim$ Age+BDI+PS+Interactions & 0.64 \\
2 & PSS $\sim$ Age+BDI+PS & 0.48 \\
3 & PSS $\sim$ Age+BDI & 0.19 \\
4 & PSS $\sim$ PS & 0.31 \\
\hline 5 & CTQTOT $\sim$ Age+BDI+PS+Interactions & 0.70 \\
6 & CTQTOT $\sim$ Age+BDI+PS & 0.39 \\
7 & CTQTOT $\sim$ Age+BDI & 0.11 \\
8 & CTQTOT $\sim$ PS & 0.29 \\
\hline 9 & PSS $\sim$ Model 1 predictors+ICV & 0.64 \\
10 & CTQTOT $\sim$ Model 1 predictors+ICV & 0.70 \\
\hline
\end{tabular}

PS: First 15 principal scores;

Interactions: age $\times$ first 5 PS and BDI $\times$ first 5 PS.
From Table I, we can see that the models including interactions between shape (PS) and confounding variables (age and BDI) have larger $R^{2}$ value than those excluding interactions. Both Models 1 and 5 achieve large $R^{2}$ values. Although BDI is highly correlated with PTSD symptoms, when we compare Models 2, 4, 6, and 8 with Models 3 and 5 that only includes age and BDI, we reach a higher $R^{2}$. This implies that shape explains more variability in the responses (PSS and CTQTOT) than BDI. Next, we focus on the significant shape principal components of each subcortical structure.

\section{TABLE II: Significant Principal Components}

\begin{tabular}{c|c|c|c} 
Response & Predictor & Sign of Coefficient & p-value \\
\hline \multirow{5}{*}{ PSS } & amygdala 4th & + & 0.0045 \\
& amygdala 5th & + & 0.0005 \\
& amygdala 6th & + & 0.006 \\
& hippocampus 2nd & + & 0.001 \\
& putamen 6th & + & 0.004 \\
\hline \multirow{5}{*}{ CTQTOT } & amygdala 2nd & + & 0.02 \\
& amygdala 11th & - & 0.0008 \\
& hippocampus 5th & - & 0.04 \\
& putamen 2nd & - & 0.01 \\
& putamen 4th & - & 0.03
\end{tabular}

Predictors are significant under significance level 0.05 .

From Table II, we see that PTSD symptoms are most correlated with subcortical shapes of the following principal components: amygdala 4th, amygdala 5th, amygdala 6th, hippocampus $2 n d$ and putamen $6 t h$. Similarly, the most significant subcortical shape changes associated with childhood traumatic experience lie in the principal directions: amygdala $2 n d$, amygdala $11 \mathrm{th}$, hippocampus $5 \mathrm{th}$, putamen $2 \mathrm{nd}$ and putamen 4th. After controlling for ICV, the principal shape components of elastic shape analysis are still found to be statistically significant. Table III presents some example significant interactions between shape and confounding patterns correlated with PTSD and traumatic experience. The full table is included in Supplementary Table 1.

TABLE III: Some Significant Interactions of Model 1 and 5

\begin{tabular}{c|c|c|c} 
Predictor for PSS & p-value & Predictor for CTQTOT & p-value \\
\hline age $\times$ amy 4th & 0.002 & age $\times$ amy $1 s t$ & $2 * 10^{-5}$ \\
BDI $\times$ amy 4th & 0.005 & BDI $\times$ amy $3 r d$ & $7 * 10^{-6}$ \\
age $\times$ hip 3rd & 0.0005 & age $\times$ hip 4th & 0.001 \\
BDI $\times$ put 1 st & 0.0007 & BDI $\times$ put 2 nd & 0.005 \\
$\ldots \ldots$ & & $\ldots \ldots$ &
\end{tabular}

amy: amygdala; hip: hippocampus; put: putamen.

Predictors are significant under significance level 0.01 .

\section{Shape Pattern}

In order to understand variations associated with different PSS and CTQTOT levels, we visualize the significant principal components for each subcortical structure. From Fig. 12, we observe that with severe PTSD symptoms:

- Left hippocampus surface moves along the positive direction of 2nd principal component, which shows a shrunken anterior end and curved body part;

- Moving along the positive direction of 4th, 5th, and 6th principal components, the left amygdala surface mainly 
deforms at the "head" end, where the central nucleus lies. The "head" end tends to indent;

- Left putamen surface's concave middle part has a larger curvature, and the end part gets thinner and sharper along the positive direction of the 6th principal component.

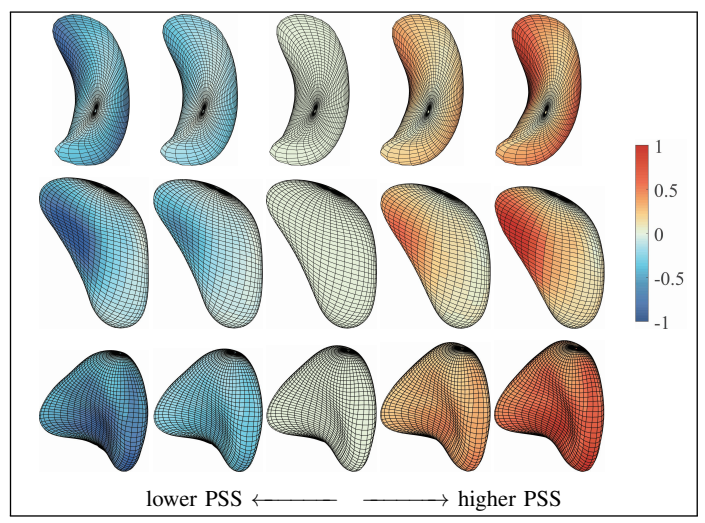

Fig. 12: Shape deformation along the most significant principal components on PTSD symptom scale. The surfaces to the left have less severe or no PTSD symptoms, and the surfaces to the right have more severe PTSD symptoms. Color indicates the small patch's relative shape difference along the direction.

Figure 13 shows that with severe childhood trauma:

- Left hippocampus surface has thinner anterior and posterior ends moving along the negative direction of 5 th principal component;

- Left amygdala surface has shrunken head end and the left side appears more flattened;

- Left putamen surface's middle part is hollower and the end part gets sharper along the negative direction of 2 nd and 4th principal component.

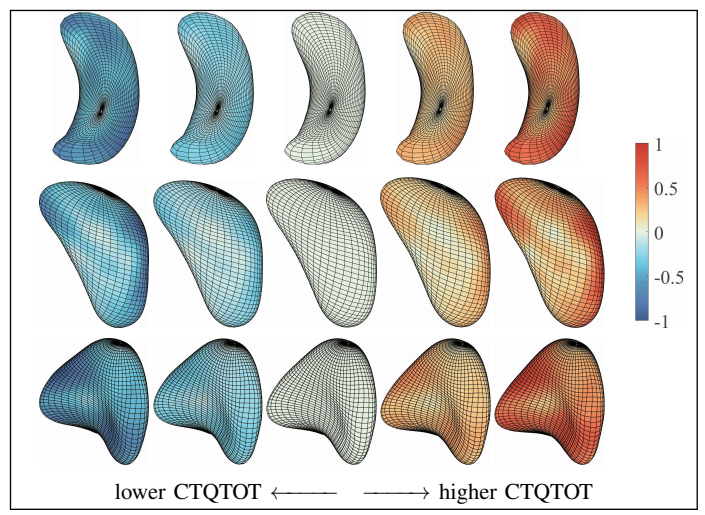

Fig. 13: Shape deformation along the most significant principal components on childhood traumatic experience inventory. The surfaces to the left have less or no childhood traumatic experience, and the surfaces to the right have more childhood traumatic experience. Color indicates the small patch's relative shape difference along the direction.

Through these visualizations, we find that the shape changes of three subcortical structures following different levels of PTSD symptoms and childhood traumatic experience are consistent. This, in turn, supports the results of regression models.
Subjects with more childhood traumatic experiences potentially have more severe PTSD, and the shape of subcortical structures deforms in the same direction. Additional displays of deformations along the most significant principal components for PSS and CTQTOT are presented in Supplementary Figs. 5 and 6. The interactive graphs to visualize shapes at different levels of PSS and CTQTOT are also presented in the Supplementary Material.

\section{Result Comparisons}

The average inter- and intra-class total point-wise distances for three subcortical structures are computed with (2) for the elastic shape analysis and vertex-wise analysis separately. The results are shown in Table IV. The inter-class distances computed under vertex-wise analysis are smaller than the intraclass distances, indicating that mis-registration errors are more significant than the differences between populations. This makes it difficult for the vertex-wise analysis to identify shape differences between healthy and PTSD groups. In contrast, the average shape differences between different groups (healthy and PTSD) are more significant than the differences within the groups with elastic surface registration. This provides strong evidence that elastic shape analysis can discover differences among subcortical shapes that are correlated with PTSD.

TABLE IV: Average total point-wise distances

\begin{tabular}{c|c|c|c|c|} 
& \multicolumn{2}{|c|}{ VERTEX-WISE } & \multicolumn{2}{c|}{ ELASTIC SHAPE } \\
\hline Structure & Inter-class & Intra-class & Inter-class & Intra-class \\
\hline amygdala & 603.1 & 608.7 & 135.8 & 130.5 \\
hippocampus & 1263.0 & 1363.9 & 317.7 & 310.7 \\
putamen & 751.3 & 823.8 & 186.4 & 182.6
\end{tabular}

To compare the two methods in the regression context, we replace the elastic shape analysis principal scores with vertexwise analysis principal scores and repeat the experiments. Table $\mathrm{V}$ lists the model adjusted $R^{2}$ values of the two methods. Regression models trained with elastic shape analysis principal scores outperform those trained with vertex-wise analysis principal scores in all six models (note that models 3 and model 7 are independent of principal scores). Besides, with the same number of principal components, those computed with the elastic shape analysis method contain more shape information (shape variation) because of the geometric properties discussed in section I.

\section{TABLE V: Adjusted $R^{2}$ of Models Under Two Methods}

\begin{tabular}{c|c|c|c|c|c}
$\#$ & Vertex-wise & Elastic Shape & $\#$ & Vertex-wise & Elastic Shape \\
\hline 1 & 0.59 & $\mathbf{0 . 6 4}$ & 5 & 0.56 & $\mathbf{0 . 7 0}$ \\
2 & 0.47 & $\mathbf{0 . 4 8}$ & 6 & 0.24 & $\mathbf{0 . 3 9}$ \\
4 & 0.23 & $\mathbf{0 . 3 1}$ & 8 & 0.18 & $\mathbf{0 . 2 9}$
\end{tabular}

From these results, we conclude that elastic shape analysis is more effective and accurate in identifying the shape differences of subcortical structures correlated with PTSD and childhood traumatic experience, when compared to the widely applied vertex-wise analysis method. 


\section{CONCLUSION}

This study uses the elastic shape analysis to compute shape summaries (mean, covariance, PCA) of subcortical data from the Grady Trauma Project (GTP). Having obtained PCA-based low-dimensional representation of shapes, we build regression models to predict PTSD clinical measures that use shapes of hippocampus, amygdala, and putamen as predictors and have considerably great predictive power. Furthermore, we localize and visualize the subcortical shape deformations related to change in PTSD severity. This tool can also provide physicians and clinicians a novel way to visualize localized changes or deformations in the brain anatomy for statistically significant shape features or principal components. Prospective studies can be carried out in larger data sizes and involving additional subcortical structures to improve predictions of PTSD clinical measures.

A key development here is the use of shape features in conjunction with additional exposure variables, such as trauma, to predict continuous clinical measures in PTSD. Such interactions are difficult to include in a vertex-wise analysis because of an inflated number of model parameters. Our predictive analysis yielded $R^{2}$ values considerably higher than the psychiatric neuroimaging literature. We explained a unique $29 \%$ of the variance in PTSD symptom severity using the principal scores above and beyond effects of age or depressive symptoms. In contrast, large collaborative meta-analyses of PTSD neuroimaging biomarkers find small effect sizes ranging from $d=0.06-0.17$ across subcortical volumes and regional cortical thickness [14], [19]. Furthermore, in comparison with the FSL FIRST analysis, elastic shape analysis produced a 5\% increase in sensitivity for the association with PTSD symptoms and a $14 \%$ increase for the association with childhood trauma exposure. This points to discovery of precision psychiatric biomarkers that rely on characterizing brain structural alterations.

We have identified an association between PTSD symptom severity and complex alterations in the hippocampus, amygdala, and putamen. With increasing PTSD symptom severity, the left hippocampus showed shrinkage of the medial wall of the head and lateral aspects of the tail, producing a more curved body shape. Although elastic shape analysis is not designed to investigate specific subfields of the hippocampus, this could be consistent with the location of CA1 and/or subiculum along the longitudinal axis of the hippocampus, consistent with prior work [6], [12]. The left amygdala showed an indentation in superior aspects located near the central subnuclei. This differs from prior literature in male veterans showing either no shape differences [12], or larger central and medial nuclei [7]. Lastly, the left putamen showed greater concavity with thinning and sharpening of the medial end, near the nucleus accumbens.

\section{ETHICS STATEMENT}

Study procedures were approved by the institutional review board of Emory University, informed consent was obtained for experimentation with human subjects, and procedures were consistent with the Declaration of Helsinki.

\section{REFERENCES}

[1] R. C. Kessler, A. Sonnega, E. Bromet, M. Hughes, and C. B. Nelson, "Posttraumatic stress disorder in the national comorbidity survey," Archives of general psychiatry, vol. 52, no. 12, pp. 1048-1060, 1995.

[2] A. Perkonigg, R. C. Kessler, S. Storz, and H.-U. Wittchen, "Traumatic events and post-traumatic stress disorder in the community: prevalence, risk factors and comorbidity," Acta psychiatrica scandinavica, vol. 101, no. 1 , pp. 46-59, 2000.

[3] C. W. Hoge, C. A. Castro, S. C. Messer, D. McGurk, D. I. Cotting, and R. L. Koffman, "Combat duty in iraq and afghanistan, mental health problems, and barriers to care," New England Journal of Medicine, vol. 351, no. 1, pp. 13-22, 2004.

[4] A. L. Mahan and K. J. Ressler, "Fear conditioning, synaptic plasticity and the amygdala: implications for posttraumatic stress disorder,' Trends in neurosciences, vol. 35, no. 1, pp. 24-35, 2012.

[5] C. B. Nemeroff, J. D. Bremner, E. B. Foa, H. S. Mayberg, C. S. North and M. B. Stein, "Posttraumatic stress disorder: a state-of-the-science review," Journal of psychiatric research, vol. 40, no. 1, pp. 1-21, 2006.

[6] L. W. Chen et al., "Smaller hippocampal ca1 subfield volume in posttraumatic stress disorder,' Depression and anxiety, vol. 35, no. 11, pp. 1018-1029, 2018.

[7] R. A. Morey et al., "Amygdala nuclei volume and shape in military veterans with posttraumatic stress disorder," Biological Psychiatry: Cognitive Neuroscience and Neuroimaging, vol. 5, no. 3, pp. 281-290, 2020.

[8] O. T. Ousdal et al., "The association of ptsd symptom severity with amygdala nuclei volumes in traumatized youths," Translational psychiatry, vol. 10, no. 1, pp. 1-10, 2020.

[9] S. H. Joshi, Q. Xie, S. Kurtek, A. Srivastava, and H. Laga, "Surface shape morphometry for hippocampal modeling in alzheimer's disease," in DICTA. IEEE, 2016, pp. 1-8.

[10] S. Kurtek et al., "Parameterization-invariant shape comparisons of anatomical surfaces," IEEE Trans. Medical Imaging, vol. 30, no. 3, pp. 849-858, 2011.

[11] I. M. Veer, N. Y. Oei, M. A. van Buchem, P. Spinhoven, B. M. Elzinga, and S. A. Rombouts, "Evidence for smaller right amygdala volumes in posttraumatic stress disorder following childhood trauma," Psychiatry Research: Neuroimaging, vol. 233, no. 3, pp. 436-442, 2015.

[12] S. Bae, C. Sheth, M. Legarreta, E. McGlade, I. K. Lyoo, and D. A. Yurgelun-Todd, "Volume and shape analysis of the hippocampus and amygdala in veterans with traumatic brain injury and posttraumatic stress disorder," Brain imaging and behavior, pp. 1-15, 2019.

[13] I. H. Jermyn, S. Kurtek, H. Laga, and A. Srivastava, "Elastic shape analysis of three-dimensional objects," Synthesis Lectures on Computer Vision, vol. 12, no. 1, pp. 1-185, 2017.

[14] M. W. Logue et al., "Smaller hippocampal volume in posttraumatic stress disorder: a multisite enigma-pgc study: subcortical volumetry results from posttraumatic stress disorder consortia," Biological psychiatry, vol. 83, no. 3, pp. 244-253, 2018.

[15] W. D. Penny, K. J. Friston, J. T. Ashburner, S. J. Kiebel, and T. E. Nichols, Statistical parametric mapping: the analysis of functional brain images. Elsevier, 2011.

[16] B. Patenaude, S. M. Smith, D. N. Kennedy, and M. Jenkinson, "A bayesian model of shape and appearance for subcortical brain segmentation," Neuroimage, vol. 56, no. 3, pp. 907-922, 2011.

[17] H. Laga, Q. Xie, I. H. Jermyn, and A. Srivastava, "Numerical inversion of srnf maps for elastic shape analysis of genus-zero surfaces," IEEE transactions on pattern analysis and machine intelligence, vol. 39, no. 12 , pp. 2451-2464, 2017.

[18] P. J. Besl and N. D. McKay, "Method for registration of 3-d shapes," in Sensor fusion IV: control paradigms and data structures, vol. 1611. International Society for Optics and Photonics, 1992, pp. 586-606.

[19] X. Wang et al., "Cortical volume abnormalities in posttraumatic stress disorder: an enigma-psychiatric genomics consortium ptsd workgroup mega-analysis," Molecular psychiatry, pp. 1-13, 2020. 